\title{
Interacting entropy-corrected holographic scalar field models in non-flat universe
}

\author{
A. Khodam-Mohammadi* and M. Malekjani ${ }^{\dagger}$ \\ Physics Department, Faculty of Science, \\ Bu-Ali Sina University, Hamedan 65178, Iran
}

\begin{abstract}
In this work we establish a correspondence between the tachyon, K-essence and dilaton scalar field models with the interacting entropy-corrected holographic dark (ECHD) model in non-flat FRW universe. The reconstruction of potentials and dynamics of these scalar fields according to the evolutionary behavior of the interacting ECHDE model are be done. It has been shown that the phantom divide can not be crossed in ECHDE tachyon model while it is achieved for ECHDE K-essence and ECHDE dilaton scenarios. At last we calculate the limiting case of interacting ECHDE model, without entropy-correction.
\end{abstract}

\footnotetext{
* E-mail: khodam@basu.ac.ir

$\dagger$ E-mail: malekjani@basu.ac.ir
} 


\section{INTRODUCTION}

The accelerated expansion of the universe is a fact which is proved by numerous cosmological observations of supernova type Ia (SNIa), cosmic microwave background (CMB) anisotropy and large scale structure (LSS). Up to now scientist believe that a component named dark energy (DE), which possesses negative pressure, is the source of this expansion. On the other hand, a curvature driven acceleration model which is called, modified gravity, has been proposed by Strobinsky [1] and Kerner [2] et al., for the first time, in 1980. Modified gravity approach suggests the gravitational alternative for unified description of inflation, dark energy and dark matter with no need of the hand insertion of extra dark components [3]. Among the DE scenarios, the cosmological constant, $\Lambda$, as vacuum energy density, with the equation of state $w_{d}=-1$ is the most well known theoretical candidate of DE. However, it suffers the so-called "fine-tuning" and "cosmic coincidence" problems [4]. The former asks why the vacuum energy density is so small [5] and the latter says why vacuum energy and dark matter nearly equal today. In order to solve these two problems, several models of dynamical dark energy models, whose equation of state is no longer a constant but evolves with time, have been proposed. The dynamical dark energy can be realized by scalar fields. Scalar field models arise in string theory and are studied as a candidates of dark energy. It includes quintessence [6], K-essence [7], phantoms [8], tachyon [9], dilaton [10], quintom [11] and so forth. The other models including Chaplygin gas [12], braneworld [13], interacting holographic DE (HDE) [14 16] and interacting agegraphic DE (ADE) models [17] have also been investigated.

Among of above models, the HDE model based on holographic principle attracted a great deal of attention in the last decade. According to the holographic principle, the number of degrees of freedom in a bounded system should be finite and has a relationship with the area of its boundary [18]. The holographic principle is a fundamental principle in quantum gravity. In quantum field theory, a short distance (UV) cut-off, $\Lambda$, is related to the long distance (IR) cut-off, $L$, due to the limit set by forming a black hole. In the other words, the zero-point energy of a system with size $L$ should not exceed the mass of a black hole with the same size. This fact directs us to $L^{3} \Lambda^{3} \leq\left(M_{p} L\right)^{3 / 2}$ [14, 19]. From this inequality, one can obtain a limit for energy density corresponding to the zero point energy and cut off $\Lambda$ as $\rho_{\Lambda} \leq M_{p}^{2} L^{-2}$ or $\rho_{\Lambda}=3 n^{2} M_{p}^{2} L^{-2}$, where $\rho_{\Lambda} \backsim \Lambda^{4}$. Here $n$ is a numerical constant 
and coefficient 3 is given for convenience. The numerical constant $n$ has been constrained by recent observation [20]. Applying the holographic principle to cosmology, the HDE as an interacting dynamical dark energy model has been constructed [16]. If we take $L$ as a size of current universe or particle horizon, the accelerated expansion of the universe can not be derived by HDE model[21]. However, in the case of future event horizon as a length scale, HDE model can derive the universe with accelerated expansion [16]. The holographic dark energy has been studied widely in the literature [22]. In the HDE scenario, the energy density depends on the entropy-area relationship of black holes in Einstein gravity. This entropy is given as $S_{\mathrm{BH}}=A /(4 G)$, where $A \sim L^{2}$ is the area of horizon. However in the context of loop quantum gravity (LQG) [23], this entropy-area relationship can be modified from the inclusion of quantum effects. The quantum corrections provided to the entropyarea relationship leads to the curvature correction in the Einstein-Hilbert action and vice versa [24, 25]. The corrected entropy is given by [26]

$$
S_{\mathrm{BH}}=\frac{A}{4 G}+\tilde{\alpha} \ln \frac{A}{4 G}+\tilde{\beta}
$$

where $\tilde{\alpha}$ and $\tilde{\beta}$ are dimensionless constants of order unity. Considering the entropy correction, the energy density of entropy-corrected holographic dark energy (ECHDE) can be given as [27]

$$
\rho_{\Lambda}=3 n^{2} M_{p}^{2} L^{-2}+\alpha L^{-4} \ln \left(M_{p}^{2} L^{2}\right)+\beta L^{-4} .
$$

The first term on right hand side is the usual holographic energy density and the other terms are due to entropy correction. The corrections make sense only at the early stage of the universe because the last two terms in Eq. (2) can be comparable to the first term only when $\mathrm{L}$ is very small. When the universe becomes large, ECHDE reduces to the ordinary HDE.

Recently, the holographic and agegraphic dark energy models have been extended regarding to the entropy correction and a thermodynamical description of the ECHDE model in a universe with spacial curvature has been done [28]. Also the correspondence of these models with some scalar fields and Chaplygin gas model have been performed [29]. Also, nowadays, many authors are interested to consider non-flat FRW universe. The tendency of a closed universe is shown in a suite CMB experiments [30]. Besides of it, the measurements of the cubic correction to the luminosity-distance of supernova measurements reveal a closed universe [31]. In accordance of all mentioned above, we prefer to consider a model including 
interacting dark matter and dark energy with a non-flat universe. We know that the scalar field models of dark energy are the effective theories of an underlying theory of dark energy. The fundamental theories including supersymmetric field theories and string/M theory can not predict the potential of scalar field uniquely. Consequently, it is meaningful to reconstruct the potential of DE model so that these scalar fields can describe the evolutionary behavior of the quantum gravity theory such as HDE model.

In this work we present a correspondence between the interacting entropy-corrected holographic dark energy (ECHDE) and the scalar filed models: K-essence, tachyon and dilaton in non-flat FRW universe. We extend the interacting ECHDE model by reconstruction the potentials and the dynamics of these scalar field models. The paper is organized as follows: In section [II, the EoS parameter of interacting ECHDE in FRW universe with spatial curvature is calculated. In sections III, IV and $\mathrm{V}$, the correspondence between the above mentioned scalar fields with interacting ECHDE in non-flat universe and reconstruction of the potentials and dynamics are presented. We concluded this work in section VI,

\section{INTERACTING ECHDE MODEL IN A NON-FLAT UNIVERSE}

Following [32], we introduce the interacting ECHDE model in non flat FRW universe. The FRW metric for non flat universe is given by

$$
d s^{2}=-d t^{2}+a^{2}(t)\left[\frac{d r^{2}}{1-k r^{2}}+r^{2}\left(d \theta^{2}+\sin ^{2} \theta d \varphi^{2}\right)\right],
$$

where $a(t)$ is the dimensionless scale factor and $k=-1,0,1$ represent the open, flat and closed universe, respectively. For the non flat FRW universe, the Friedmann equation can be written as

$$
H^{2}+\frac{k}{a^{2}}=\frac{1}{3 M_{p}^{2}}\left[\rho_{\Lambda}+\rho_{m}\right],
$$

where $M_{p}=(8 \pi G)^{-1 / 2}$ is modified Planck mass, $H$ is the Hubble parameter and $\rho_{\Lambda}$ and $\rho_{m}$ are the energy densities of dark energy and dark matter, respectively. With the definition of dimensionless parameters $\Omega_{m}=\rho_{m} / \rho_{c}, \Omega_{\Lambda}=\rho_{\Lambda} / \rho_{c}$ and $\Omega_{k}=k /(a H)^{2}$, corresponding to energy density of matter, dark energy and curvature, respectively, the Friedmann equation (4) is written as

$$
1+\Omega_{k}=\Omega_{\Lambda}+\Omega_{m}
$$


Here $\rho_{c}=3 H^{2} M_{p}^{2}$ is the critical density. By assuming an interaction between dark matter and dark energy, the energy conservation equation is separated for two energy density component, of matter and dark energy as

$$
\begin{aligned}
& \dot{\rho}_{\Lambda}+3 H \rho_{\Lambda}\left(1+w_{\Lambda}\right)=-Q, \\
& \dot{\rho}_{m}+3 H \rho_{m}=Q
\end{aligned}
$$

where $w_{\Lambda}$ is EOS parameter of DE. The term $Q=3 b^{2} H\left(\rho_{m}+\rho_{\Lambda}\right)$ is an energy exchange term and $b^{2}$ is a coupling parameter between dark matter and dark energy. However the nature of dark matter and dark energy remains unknown, different lagrangian have been proposed to generate this interaction term [33]. Also above expression for $Q$ may look purely phenomenologically. It is worthwhile to mention that this phenomenological description of interaction may be proved with some recent observations in galaxy clusters [34], and SNIa, CMB, LSS, and age constraints [35]. Three forms of interaction has been proposed by authors. Also the dynamic of interacting dark energy models have been studied. At first this expression was used in the study of the suitable coupling between a quintessence scalar field and a pressureless cold dark matter [36].

The energy density of ECHDE (21) contains a length scale $L$ as a future event horizon. Since we have

$$
L=a(t) r(t)
$$

where $a(t)$ is scale factor and $r(t)$ is relevant to the future event horizon of the observable universe. The size of future event horizon can be defined as

$$
R_{h}=a(t) \int_{t}^{\infty} \frac{d t^{\prime}}{a\left(t^{\prime}\right)}=a(t) \int_{0}^{r_{1}} \frac{d r}{\sqrt{1-k r^{2}}}
$$

Giving the fact that

$$
\int_{0}^{r_{1}} \frac{d r}{\sqrt{1-k r^{2}}}=\frac{1}{\sqrt{|k|}} \operatorname{sinn}^{-1}\left(\sqrt{|k|} r_{1}\right)= \begin{cases}\sin ^{-1}\left(r_{1}\right), & k=+1 \\ r_{1}, & k=0 \\ \sinh ^{-1}\left(r_{1}\right), & k=-1\end{cases}
$$

we can easily derive

$$
L=a(t) \frac{\operatorname{sinn}(\sqrt{|k|} y)}{\sqrt{|k|}}, \quad y=\frac{R_{h}}{a(t)},
$$


Considering the energy density of ECHDE (2), the following relation can be obtained

$$
H L=\sqrt{\frac{3 n^{2} m_{p}^{2}+\alpha L^{-2} \ln \left(m_{p}^{2} L^{2}\right)+\beta L^{-2}}{3 m_{p}^{2} \Omega_{\Lambda}}} .
$$

By differentiating Eq.(11) with respect to time, using Eq.(12) and the fact that $\dot{y}=a$ from Eq. (9), we obtain

$$
\dot{L}=\sqrt{\frac{3 n^{2} m_{p}^{2}+\alpha L^{-2} \ln \left(m_{p}^{2} L^{2}\right)+\beta L^{-2}}{3 m_{p}^{2} \Omega_{\Lambda}}}-\operatorname{cosn}(\sqrt{|k|} y),
$$

where $\operatorname{cosn}(x)$ is the derivative of $\operatorname{sinn}(x)$ and we also have

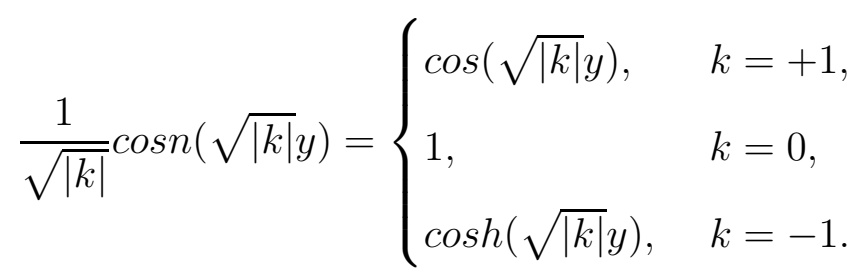

Taking derivative of Eq.(2) with respect to time and using Eq.(13), we get

$$
\begin{aligned}
\dot{\rho}_{\Lambda}= & \left(2 \alpha L^{-5}-4 \alpha L^{-5} \ln \left(M_{p}^{2} L^{2}\right)-4 \beta L^{-5}-6 n^{2} M_{p}^{2} L^{-3}\right) \\
& \times\left[\sqrt{\frac{3 n^{2} M_{p}^{2}+\alpha L^{-2} \ln \left(M_{p}^{2} L^{2}\right)+\beta L^{-2}}{3 M_{p}^{2} \Omega_{\Lambda}}}-\operatorname{cosn}(\sqrt{|k|} y)\right] .
\end{aligned}
$$

Inserting Eq. (15) in Eq. (66), the EoS parameter of interacting ECHDE model can be obtained as

$$
w_{\Lambda}=-1-\frac{2\left(-2 D_{L}+3 n^{2} M_{p}^{2} L^{2}+\alpha\right)\left(1-\sqrt{\frac{3 M_{p}^{2} L^{2} \Omega_{\Lambda}}{D_{L}}} \operatorname{cosn}(\sqrt{|k|} y)\right)}{3 D_{L}}-\frac{b^{2}\left(1+\Omega_{k}\right)}{\Omega_{\Lambda}}
$$

where we define the parameter $D_{L}$ as

$$
D_{L}=3 n^{2} M_{p}^{2} L^{2}+\alpha \ln M_{p}^{2} L^{2}+\beta=L^{4} \rho_{\Lambda}
$$

As we can see from (16), the phantom divide $\left(w_{\Lambda}<-1\right)$ may cross for a wide range of parameters $n, b, \alpha$ and $\beta$. Setting $\alpha=\beta=0$ in Eq.(16), we have $D_{L}=3 n^{2} M_{p}^{2} L^{2}$, and the EoS parameter of interacting holographic dark energy in non flat universe can easily obtained as

$$
w_{\Lambda}=-\frac{1}{3}-\frac{2}{3} \frac{\sqrt{\Omega_{\Lambda}}}{n} \operatorname{cosn}(\sqrt{|k|} y)-\frac{b^{2}\left(1+\Omega_{k}\right)}{\Omega_{\Lambda}} .
$$

In the case of HDE without interaction, $b=0$, the last term of Eq.(18) will be zero which is the same as Rel. (23) of Ref. [37]. 


\section{INTERACTING ECHDE TACHYON MODEL}

Tachyon is an unstable field which has been described in string theory through its role in the Dirac-Born-Infeld (DBI) action to describe the D-bran action [38]. The effective Lagrangian for the tachyon field is given by

$$
\mathcal{L}=-V(\phi) \sqrt{1-g^{\mu \nu} \partial_{\mu} \phi \partial_{\nu} \phi}
$$

where $V(\phi)$ is the potential of tachyon. The energy density and pressure for the tachyon field are as following [38]

$$
\begin{gathered}
\rho_{\phi}=\frac{V(\phi)}{\sqrt{1-\dot{\phi}^{2}}}, \\
p_{\phi}=-V(\phi) \sqrt{1-\dot{\phi}^{2}} .
\end{gathered}
$$

The EoS parameter of tachyon can be obtained as

$$
w_{\phi}=\frac{p_{\phi}}{\rho_{\phi}}=\dot{\phi}^{2}-1
$$

Now we can establish the correspondence between the interacting ECHDE scenario and the tachyon scalar field model. By comparing Eqs.(2) and (19), we have

$$
V(\phi)=\frac{D_{L}}{L^{4}} \sqrt{1-\dot{\phi}^{2}}
$$

while equating Eqs.(16) and (21) , give us $\dot{\phi}$ as

$$
\dot{\phi}^{2}=1+w_{\Lambda}=\frac{2\left(2 D_{L}-3 n^{2} M_{p}^{2} L^{2}-\alpha\right)\left(1-\sqrt{\frac{3 M_{p}^{2} L^{2} \Omega_{\Lambda}}{D_{L}}} \operatorname{cosn}(\sqrt{|k|} y)\right)}{3 D_{L}}-\frac{b^{2}\left(1+\Omega_{k}\right)}{\Omega_{\Lambda}} .
$$

Using the Eqs.(22) and (23), we can write the potential of tachyon as

$$
\begin{aligned}
V(\phi)= & \frac{D_{L}}{L^{4}} \sqrt{-w_{\Lambda}}= \\
& \frac{D_{L}}{L^{4}} \sqrt{1-\frac{2\left(2 D_{L}-3 n^{2} M_{p}^{2} L^{2}-\alpha\right)\left(1-\sqrt{\frac{3 M_{p}^{2} L^{2} \Omega_{\Lambda}}{D_{L}}} \operatorname{cosn}(\sqrt{|k|} y)\right)}{3 D_{L}}+\frac{b^{2}\left(1+\Omega_{k}\right)}{\Omega_{\Lambda}} .}
\end{aligned}
$$

As we can see from Eqs.(23) and (24), the kinetic energy $\dot{\phi}^{2}$ and potential $V(\phi)$ may exist provided that

$$
-1 \leq w_{\Lambda} \leq 0
$$


This condition implies that the phantom divide can not be crossed in a universe with an accelerated expansion.

In the limiting case of $\alpha=\beta=0$, the kinetic energy and potential for usual holographic tachyon dark energy without the interaction term $(b=0)$ can be obtained exactly the same as ones in Ref. [37] as:

$$
\begin{aligned}
& \dot{\phi}=\sqrt{\frac{2}{3}} \sqrt{1-\frac{\sqrt{\Omega_{\Lambda}}}{n} \operatorname{cosn}(\sqrt{|k|} y)}, \\
& V(\phi)=\frac{\sqrt{3} n^{2} M_{p}^{2}}{L^{2}} \sqrt{1+2 \frac{\sqrt{\Omega_{\Lambda}}}{n} \operatorname{cosn}(\sqrt{|k|} y)} .
\end{aligned}
$$

Using $\dot{\phi}=\phi^{\prime} H$ and Eq.(23), we get

$$
\phi^{\prime}=\frac{1}{H} \sqrt{\frac{2\left(2 D_{L}-3 n^{2} M_{p}^{2} L^{2}-\alpha\right)\left(1-\sqrt{\frac{3 M_{p}^{2} L^{2} \Omega_{\Lambda}}{D_{L}}} \operatorname{cosn}(\sqrt{|k|} y)\right)}{3 D_{L}}-\frac{b^{2}\left(1+\Omega_{k}\right)}{\Omega_{\Lambda}}}
$$

Hence, we can find the evolutionary form of the tachyon scalar field as follows

$$
\begin{aligned}
& \phi(a)-\phi\left(a_{0}\right)= \\
& \int_{a_{0}}^{a} \frac{1}{a H} \sqrt{\frac{\left(2 D_{L}-3 n^{2} M_{p}^{2} L^{2}-\alpha\right)\left(1-\sqrt{\frac{3 M_{p}^{2} L^{2} \Omega_{\Lambda}}{D_{L}}} \operatorname{cosn}(\sqrt{|k|} y)\right)}{D_{L}}-\frac{b^{2}\left(1+\Omega_{k}\right)}{\Omega_{\Lambda}} d a .}
\end{aligned}
$$

Here $a_{0}$ is the present value of the scale factor. Here, we have established an interacting entropy-corrected holographic tachyon dark energy model and reconstructed the potential and the dynamics of the tachyon field. Setting $\alpha=\beta=0$ and $b=0$ in Eqs. (28) and (29), one can easily see that in the case of non-interacting HDE, the evolutionary form of tachyon field in non-flat universe can be obtained as follows

$$
\phi(a)-\phi\left(a_{0}\right)=\sqrt{\frac{2}{3}} \int_{a_{0}}^{a} \frac{1}{a H} \sqrt{1-\frac{\sqrt{\Omega_{\Lambda}}}{n} \operatorname{cosn}(\sqrt{|k|} y)} d a
$$

\section{INTERACTING ECHDE K-ESSENCE MODEL}

The K-essence scalar field model can explain the late time acceleration of the universe. The general scalar field action for K-essence model as a function of $\phi$ and $\chi=\dot{\phi}^{2} / 2$ is given by [39]

$$
S=\int d^{4} x \sqrt{-g} p(\phi, \chi),
$$


where the Lagrangian density $p(\phi, \chi)$ relates to a pressure density and energy density through the following equations:

$$
\begin{gathered}
p(\phi, \chi)=f(\phi)\left(-\chi+\chi^{2}\right), \\
\rho(\phi, \chi)=f(\phi)\left(-\chi+3 \chi^{2}\right) .
\end{gathered}
$$

Hence, the EoS parameter of K-essence scalar field is obtained as

$$
\omega_{K}=\frac{p(\phi, \chi)}{\rho(\phi, \chi)}=\frac{\chi-1}{3 \chi-1} .
$$

In order to consider the K-essence field as a description of the interacting ECHDE density, we establish the correspondence between the K-essence EoS parameter, $w_{K}$, and the interacting ECHDE EoS parameter, $w_{\Lambda}$. Equating Eq.(33) with Eq.(2), we have

$$
f(\phi)=\frac{D(L)}{L^{4}}\left[\frac{\left(1-3 w_{\Lambda}\right)^{2}}{2\left(1-w_{\Lambda}\right)}\right]
$$

while by equating Eq.(34) with Eq.(16), we can find the expression for $\chi$ as

$$
\begin{aligned}
& \chi=\frac{w_{\Lambda}-1}{3 w_{\Lambda}-1}= \\
& \frac{1}{3}\left[\frac{-6+2\left(2 D_{L}-3 n^{2} M_{p}^{2} L^{2}-\alpha\right)\left(1-\sqrt{\frac{3 M_{p}^{2} L^{2} \Omega_{\Lambda}}{D_{L}}} \operatorname{cosn}(\sqrt{|k|} y)\right) D_{L}^{-1}-3 b^{2}\left(1+\Omega_{k}\right) \Omega_{\Lambda}^{-1}}{-4+2\left(2 D_{L}-3 n^{2} M_{p}^{2} L^{2}-\alpha\right)\left(1-\sqrt{\frac{3 M_{p}^{2} L^{2} \Omega_{\Lambda}}{D_{L}}} \operatorname{cosn}(\sqrt{|k|} y)\right) D_{L}^{-1}-3 b^{2}\left(1+\Omega_{k}\right) \Omega_{\Lambda}^{-1}}\right]
\end{aligned}
$$

Using $\dot{\phi}^{2}=2 \chi$, and $\dot{\phi}=\phi^{\prime} H$, we get

$$
\begin{aligned}
& \phi^{\prime}=\frac{1}{H} \sqrt{\frac{2}{3}} \sqrt{1+\frac{2}{1-3 w_{\Lambda}}}=\frac{1}{H} \sqrt{\frac{2}{3}} \times \\
& \sqrt{\frac{-6+2\left(2 D_{L}-3 n^{2} M_{p}^{2} L^{2}-\alpha\right)\left(1-\sqrt{\frac{3 M_{p}^{2} L^{2} \Omega_{\Lambda}}{D_{L}}}\right.}{-4+2\left(2 D_{L}-3 n^{2} M_{p}^{2} L^{2}-\alpha\right)\left(1-\sqrt{\frac{3 M_{p}^{2} L^{2} \Omega_{\Lambda}}{D_{L}}}\right.} \cos (\sqrt{|k|}(\sqrt{|k|} y)) D_{L}^{-1}-3 b^{2}\left(1+\Omega_{k}\right) \Omega_{\Lambda}^{-1}-3 b^{2}\left(1+\Omega_{k}\right) \Omega_{\Lambda}^{-1}} .
\end{aligned}
$$

Integrating Eq.(37), we obtain the evolutionary form of the K-essence scalar field as

$$
\begin{aligned}
& \phi(a)-\phi\left(a_{0}\right)=\sqrt{\frac{2}{3}} \int_{a_{0}}^{a} \frac{d a}{a H} \times \\
& \sqrt{\left.\frac{-6+2\left(2 D_{L}-3 n^{2} M_{p}^{2} L^{2}-\alpha\right)\left(1-\sqrt{\frac{3 M_{p}^{2} L^{2} \Omega_{\Lambda}}{D_{L}}}\right.}{-4+2\left(2 D_{L}-3 n^{2} M_{p}^{2} L^{2}-\alpha\right)\left(1-\sqrt{\frac{3 M_{p}^{2} L^{2} \Omega_{\Lambda}}{D_{L}}}\right.} \operatorname{cosn}(\sqrt{|k|} y)\right) D_{L}^{-1}-3 b^{2}\left(1+\Omega_{k}\right) \Omega_{\Lambda}^{-1}} .
\end{aligned}
$$


We see from Eq. (37), the kinetic energy $\dot{\phi}^{2}$ may exist provided that

$$
w_{\Lambda} \leq 1
$$

This condition implies that the phantom divide can be crossed in a universe with an accelerated expansion.

In the limiting case of $\alpha=\beta=0$ and $b=0$, Eqs. (37), (37) and (38) reduce to the following simple form for non-interacting holographic K-essence model in non-flat universe.

$$
\begin{gathered}
\chi=\frac{1}{3} \frac{2+\frac{\sqrt{\Omega_{\Lambda}}}{n} \operatorname{cosn}(\sqrt{|k|} y)}{1+\frac{\sqrt{\Omega_{\Lambda}}}{n} \operatorname{cosn}(\sqrt{|k|} y)} \\
\phi^{\prime}=\sqrt{\frac{2}{3}} \frac{1}{H} \sqrt{\frac{2+\frac{\sqrt{\Omega_{\Lambda}}}{n} \operatorname{cosn}(\sqrt{|k|} y)}{1+\frac{\sqrt{\Omega_{\Lambda}}}{n} \operatorname{cosn}(\sqrt{|k|} y)}} \\
\phi(a)-\phi\left(a_{0}\right)=\sqrt{\frac{2}{3}} \int_{a_{0}}^{a} \frac{1}{a H} \sqrt{\frac{2+\frac{\sqrt{\Omega_{\Lambda}}}{n} \operatorname{cosn}(\sqrt{|k|} y)}{1+\frac{\sqrt{\Omega_{\Lambda}}}{n} \operatorname{cosn}(\sqrt{|k|} y)}} d a
\end{gathered}
$$

\section{INTERACTING ECHDE DILATON MODEL}

The dilaton scalar field is originated from the lower limit of string theory [40]. The dilaton filed is described by the effective Lagrangian density as

$$
p_{D}=-\chi+c e^{\lambda \phi} \chi^{2}
$$

where $c$ and $\lambda$ are positive constant. Considering the dilaton field as a source of the energymomentum tensor in Einstein equations, one can find that the Lagrangian density corresponds to the pressure of the scalar field and the energy density of dilaton field is also obtained as

$$
\rho_{D}=-\chi+3 c e^{\lambda \phi} \chi^{2}
$$

where $2 \chi=\dot{\phi}^{2}$. The negative coefficient of the kinematic term of the dilaton field in Einstein frame makes a phantom like behavior for dilaton field. The EoS parameter of dilaton is given by

$$
\omega_{D}=\frac{p_{D}}{\rho_{D}}=\frac{-1+c e^{\lambda \phi} \chi}{-1+3 c e^{\lambda \phi} \chi} .
$$


In order to consider the dilaton field as a description of the interacting ECHDE density, we establish the correspondence between the dilaton EoS parameter, $w_{D}$, and the EoS parameter $w_{\Lambda}$ of interacting ECHDE. By equating Eq.(45) with Eq.(16), we can find

$$
\begin{aligned}
& c e^{\lambda \phi} \chi=\frac{w_{\Lambda}-1}{3 w_{\Lambda}-1}= \\
& \frac{1}{3}\left[\frac{-6+2\left(2 D_{L}-3 n^{2} M_{p}^{2} L^{2}-\alpha\right)\left(1-\sqrt{\frac{3 M_{p}^{2} L^{2} \Omega_{\Lambda}}{D_{L}}} \operatorname{cosn}(\sqrt{|k|} y)\right) D_{L}^{-1}-3 b^{2}\left(1+\Omega_{k}\right) \Omega_{\Lambda}^{-1}}{-4+2\left(2 D_{L}-3 n^{2} M_{p}^{2} L^{2}-\alpha\right)\left(1-\sqrt{\frac{3 M_{p}^{2} L^{2} \Omega_{\Lambda}}{D_{L}}} \operatorname{cosn}(\sqrt{|k|} y)\right) D_{L}^{-1}-3 b^{2}\left(1+\Omega_{k}\right) \Omega_{\Lambda}^{-1}}\right] .
\end{aligned}
$$

using $\chi=\dot{\phi}^{2} / 2$ and $\dot{\phi}=\phi^{\prime} / H$, one can rewrite (46) with respect to $\phi$ as

$$
\begin{aligned}
& \frac{d}{d \ln a} e^{\lambda \phi / 2}=\frac{1}{H} \frac{\lambda}{\sqrt{6 c}} \sqrt{1+\frac{2}{1-3 w_{\Lambda}}}=\frac{1}{H} \frac{\lambda}{\sqrt{6 c}} \times \\
& \sqrt{\frac{-6+2\left(2 D_{L}-3 n^{2} M_{p}^{2} L^{2}-\alpha\right)\left(1-\sqrt{\frac{3 M_{p}^{2} L^{2} \Omega_{\Lambda}}{D_{L}}} \operatorname{cosn}(\sqrt{|k|} y)\right) D_{L}^{-1}-3 b^{2}\left(1+\Omega_{k}\right) \Omega_{\Lambda}^{-1}}{-4+2\left(2 D_{L}-3 n^{2} M_{p}^{2} L^{2}-\alpha\right)\left(1-\sqrt{\frac{3 M_{p}^{2} L^{2} \Omega_{\Lambda}}{D_{L}}} \operatorname{cosn}(\sqrt{|k|} y)\right) D_{L}^{-1}-3 b^{2}\left(1+\Omega_{k}\right) \Omega_{\Lambda}^{-1}}},
\end{aligned}
$$

Exactly the same as Sec. IV] from Eq. (46), one can see that the kinetic energy may exist provided that $w_{\Lambda} \leq 1$ which implies that the phantom divide can be crossed in a universe with an accelerated expansion.

Finally the evolutionary form of the dilaton scalar field is written as

$$
\begin{aligned}
& \phi(a)=\frac{2}{\lambda} \ln \left\{e^{\lambda \phi\left(a_{0}\right) / 2}+\frac{\lambda}{\sqrt{6 c}} \int_{a_{0}}^{a} \frac{d a}{a H} \times\right. \\
& \sqrt{\frac{-6+2\left(2 D_{L}-3 n^{2} M_{p}^{2} L^{2}-\alpha\right)\left(1-\sqrt{\frac{3 M_{p}^{2} L^{2} \Omega_{\Lambda}}{D_{L}}}\right.}{-4+2\left(2 D_{L}-3 n^{2} M_{p}^{2} L^{2}-\alpha\right)\left(1-\sqrt{\frac{3 M_{p}^{2} L^{2} \Omega_{\Lambda}}{D_{L}}} \operatorname{cosn}(\sqrt{|k|} y)\right) D_{L}^{-1}-3 b^{2}\left(1+\Omega_{k}\right) \Omega_{\Lambda}^{-1}}} . \\
& \sqrt{|k|} y)) D_{L}^{-1}-3 b^{2}\left(1+\Omega_{k}\right) \Omega_{\Lambda}^{-1}
\end{aligned}
$$

In the limiting case of $\alpha=\beta=0$ and $b=0$, Eq.(48) reduce to the following simple form for non-interacting holographic dilaton model in non-flat universe. Therefore the evolutionary form of the dilaton scalar field can be obtained as

$$
\phi(a)=\frac{2}{\lambda} \ln \left[e^{\lambda \phi\left(a_{0}\right) / 2}+\frac{\lambda}{\sqrt{6 c}} \int_{a_{0}}^{a} \frac{1}{a H} \sqrt{\frac{2+\frac{\sqrt{\Omega_{\Lambda}}}{n} \operatorname{cosn}(\sqrt{|k|} y)}{1+\frac{\sqrt{\Omega_{\Lambda}}}{n} \operatorname{cosn}(\sqrt{|k|} y)}} d a\right] .
$$

\section{CONCLUSION}

Including the quantum correction in the entropy-area relation, we studied the interacting ECHDE model in non-flat FRW universe. As is well known, the scalar field models 
are the effective description of an underlying theory of dark energy. We established the correspondence between tachyon, K-essence and dilaton scalar field models with interacting ECHDE model. By this correspondence, the scalar fields may be reconstructed so that they can describe the evolutionary behavior of interacting ECHDE model. The reconstruction of potentials and dynamics of these scalar fields which describe the tachyon, K-essence and dilaton cosmology was obtained. It has been shown that the phantom divide can not be crossed in ECHDE tachyon model while it is achieved for ECHDE K-essence and ECHDE dilaton scenarios. By putting $\alpha=\beta=0$, the EoS parameter of HDE, potentials and the evolutionary form of HDE scalar field models have been calculated.

[1] A. A. Starobinsky, Phys. Lett. B 91, 99 (1980).

[2] R. Kerner, Gen. Rel. Gravit. 14, 453 (1982); J. P. Duruisseau, and R. Kerner, Class. Quantum Grav. 3, 817 (1986).

[3] S. Nojiri and S. D. Odintsov, Gen. Rel. Grav. 38,1285 (2006); G. Cognola, E. Elizalde, S. Nojiri, S.D. Odintsov, and S. Zerbini, JCAP 0502, 010 (2005); S. Nojiri, and S.D. Odintsov, Int. J. Geom. Meth. Mod. Phys 4, 115 (2007); S. Nojiri, and S. D. Odintsov, Phys. Rev. D 68, 123512 (2003); A. Khodam-Mohammadi, P. majari, and M. Malekjani, Astrophys. Space Sci., 331, 673 (2011).

[4] E.J. Copeland, M. Sami, and S. Tsujikawa, Int. J. Mod. Phys. D 15, 1753 (2006).

[5] S. Weinberg, Rev. Mod. Phys. 61, 1 (1989).

[6] C. Wetterich, Nucl. Phys. B 302, 668 (1988); B. Ratra, and J. Peebles, Phys. Rev. D 37, 321 (1988).

[7] T. Chiba, T. Okabe, and M. Yamaguchi, Phys. Rev. D 62, 023511 (2000); C. ArmendárizPicón, V. Mukhanov, and P.J. Steinhardt, Phys. Rev. Lett. 85, 4438 (2000); C. ArmendárizPicón, V. Mukhanov, and P.J. Steinhardt, Phys. Rev. D 63, 103510 (2001).

[8] R.R. Caldwell, Phys. Lett. B 545, 23 (2002); S. Nojiri, and S.D. Odintsov, Phys. Lett. B 562, 147 (2003); S. Nojiri, and S.D. Odintsov, Phys. Lett. B 565, 1 (2003).

[9] A. Sen, J. High Energy Phys. 10, 008 (1999); E.A. Bergshoeff, M. de Roo, T.C. de Wit, E. Eyras, and S. Panda, J. High Energy Phys. 05, 009 (2000); A. Sen, J. High Energy Phys. 04, 048 (2002); A. Sen, J. High Energy Phys. 07, 065 (2002); T. Padmanabhan, Phys. Rev. D 
66, 021301 (2002); T. Padmanabhan, and T.R. Choudhury, Phys. Rev. D 66, 081301 (2002); L.R.W. Abramo, and F. Finelli, Phys. Lett. B 575, 165 (2003).

[10] M. Gasperini, F. Piazza, and G. Veneziano, Phys. Rev. D 65, 023508 (2002); N. ArkaniHamed, P. Creminelli, S. Mukohyama, and M. Zaldarriaga, J. Cosmol. Astropart. Phys. 04, 001 (2004); F. Piazza, and S. Tsujikawa, J. Cosmol. Astropart. Phys. 07, 004 (2004).

[11] E. Elizalde, S. Nojiri, and S.D. Odinstov, Phys. Rev. D 70, 043539 (2004); S. Nojiri, S.D. Odintsov, and S. Tsujikawa, Phys. Rev. D 71, 063004 (2005); A. Anisimov, E. Babichev, and A. Vikman, J. Cosmol. Astropart. Phys. 06, 006 (2005).

[12] A. Kamenshchik, U. Moschella, and V. Pasquier, Phys. Lett. B 511, 265 (2001); M.C. Bento, O. Bertolami, and A.A. Sen, Phys. Rev. D 66, 043507 (2002).

[13] C. Deffayet, G.R. Dvali, and G. Gabadadze, Phys. Rev. D 65, 044023 (2002); V. Sahni, and Y. Shtanov, J. Cosmol. Astropart. Phys. 11, 014 (2003).

[14] A. Cohen, D. Kaplan, and A. Nelson, Phys. Rev. Lett. 82, 4971 (1999).

[15] P. Horava, and D.Minic, Phys. Rev. Lett. 85, 1610 (2000); S. Thomas, Phys. Rev. Lett. 89, $081301(2002)$.

[16] M. Li, Phys. Lett. B 603, 1(2004).

[17] R.G. Cai, Phys. Lett. B 657, 228 (2007); H. Wei, and R.G. Cai, Phys. Lett. B 660, 113 (2008).

[18] G. 't Hooft, gr-qc/9310026]; L. Susskind, J. Math. Phys. 36, 6377 (1995).

[19] J. D. Bekenstein, Phys. Rev. D 7 (1973) 2333; S. W. Hawking, Comm. Math. Phys. 43, 199 (1975); S. W. Hawking, Phys. Rev. D 13, 191 (1976); J. D. bekenstein, Phys. Rev. D 23 , 287 (1981).

[20] M. Li, X. D. Li, S. Wang, Y. Wang, and X. Zhang, arXiv:0910.3855; M. Li, X. D. Li, S. Wang, Y. Wang, and X. Zhang, J. Cosmol. Astropart. Phys. 06, 036 (2009).

[21] S. D. H. Hsu, Phys. Lett. B 669, 275 (2008).

[22] E. Elizalde, S. Nojiri, S.D. Odintsov, and P. Wang, Phys. Rev. D 71, 103504 (2005); M. R. Setare, Phys. Lett. B 648, 329 (2007); M. R. Setare, and M. Jamil, JCAP, 02, 010 (2010); K. Karami, and J. Fehri, Phys. Lett. B, 686, 216 (2010); A. Sheykhi, Phys. Lett. B 681, 205 (2009); M. R. Setare, and S. Shafei, JCAP 09, 011 (2006); M. R. Setare, Phys. Lett. B 644, 99 (2007); M. R. Setare, and E. C. Vagenas, Phys. Lett. B 666, 111 (2008); M. R. Setare, Phys. Lett. B 642, 421 (2006); M. R. Setare, Phys. Lett. B 654, 1 (2007); M. R. Setare, Eur. Phys. J. C 52, 689 (2007). 
[23] C. Rovelli, Phys. Rev. Lett. 77, 3288 (1996); A. Ashtekar, J. Baez, A. Corichi and K. Krasnov, Phys. Rev. Lett. 80, 904 (1998); R. K. Kaul and P. Majumdar, Phys. Rev. Lett. 84, 5255 (2000); A. Ghosh and P. Mitra, Phys. Rev. D 71, 027502 (2005); M. Domagala and J. Lewandowski, Class. Quant. Grav. 21, 5233 (2004); K. A. Meissner, Class. Quant. Grav. 21, 5245 (2004); A. J. M. Medved, Class. Quant. Grav. 22, 133 (2005); A. J. M. Medved and E. C. Vagenas, Phys. Rev. D 70, 124021 (2004).

[24] T. Zhu and J-R. Ren, Eur. Phys. J. C 62, 413 (2009); R-G. Cai et al, Class. Quant. Grav. 26, 155018 (2009).

[25] S. Nojiri and S.D. Odintsov, Int. J. Mod. Phys. A 16, 3273 (2001).

[26] R. Banerjee, and B.R. Majhi, Phys. Lett. B 662, 62 (2008); R. Banerjee, and B.R. Majhi, JHEP. 06, 095 (2008); R. Banerjee, and S.K. Modak, J. High Energy Phys. 05, 063 (2009); B.R. Majhi, Phys. Rev. D 79, 044005 (2009); S.K. Modak, Phys. Lett. B 671, 167 (2009); M. Jamil, and M.U. Farooq, J. Cosmol. Astropart. Phys. 03, 001 (2010); H.M. Sadjadi, and M. Jamil, EPL, 92, 69001 (2010) arXiv:1002.3588; S.-W. Wei, Y.-X. Liu, Y.-Q. Wang, and H. Guo, arXiv:1002.1550; D.A. Easson, P.H. Frampton, and G.F. Smoot, arXiv:1003.1528].

[27] H. Wei, Commun. Theor. Phys. 52, 743 (2009).

[28] M. Jamil, A. Sheykhi, M. U. Farooq, Int. J. Mod. Phys. D 19, 1831 (2010).

[29] M. Jamil and M. U. Farooq, JCAP 03, 001 (2010); M. Jamil, and A. Sheykhi, Int. J. Theor. Phys., 50, 625 (2011); M. Malekjani, and A. Khodam-Mohammadi, [arxive:1004.1017].

[30] J. L. Sievers, et al., Astrophys. J. 591, 599 (2003); C.B. Netterfield, et al., Astrophys. J. 571, 604 (2002); A. Benoit, et al., Astron. Astrophys. 399, L25 (2003); A. Benoit, et al., Astron. Astrophys. 399, L19 (2003).

[31] R. R. Caldwell, and M. Kamionkowski, JCAP 0409, 009 (2004); B. Wang, Y.G. Gong, and R.K. Su, Phys. Lett. B 605, 9 (2005).

[32] M. U. Farooq, M. A. Rashid, and M. Jamil, Int. J. Theor. Phys., 49, 2278 (2010).

[33] S. Tsujikawa and M. Sami, Phys. Lett. B 603, 113 (2004).

[34] O. Bertolami, F. Gil Pedro, and M. le delliou, Phys. Lett. B 654, 165 (2007).

[35] B. wang, Ch.-Y. Lin, and E. Aballa, Phys. Lett. B 637, 357 (2006); B. wang, J. zang, Ch.-Y. Lin, E. Abdalla, and S. Micheletti, Gen. Rel. Grav. 41, 2839 (2009).

[36] L. Amendola, Phys. Rev. D 60, 043501 (1999);L. Amendola, Phys. Rev. D 62, 043501 (2000);L. Amendola, and C. Quercellini, Phys. Rev. D 68, 023514 (2003). 
[37] M. R. Setare, Phys.Lett.B 653, 116 (2007).

[38] A. Sen, JHEP 0204, 048 (2002);A. Sen, JHEP 0207, 065 (2002); A. Sen, Mod. Phys. Lett. A 17, 1797 (2002); A. Sen, [hep- th/0312153];A. Sen, JHEP 9910, 008 (1999); E. A. Bergshoeff, M. de Roo, T. C. de Wit, E. Eyras, and S. Panda, JHEP 0005, 009 (2000); J. Kluson, Phys. Rev. D 62, 126003 (2000); D. Kutasov and V. Niarchos, Nucl. Phys. B 666, 56, (2003).

[39] T. Chiba et al., Phys. Rev. D 62, 023511 (2000); C. Armendariz-Picon et al., Phys. Rev. Lett 85, 4438 (2000).

[40] F. Piazza and S. Tsujikawa, JCAP 0407, 004 (2004). 